\title{
Catálogo selectivo de las obras musicales de Vicente Bianchi Alarcón
}

\author{
A selective catalogue of musical Works by \\ Vicente Bianchi Alarcón
}

\author{
por \\ Julio Garrido Letelier \\ Facultad de Artes. Universidad de Chile \\ julio.garrido.1@uchile.cl
}

El presente catálogo se ha elaborado a base de la lista de obras confeccionada por Rodrigo Torres y publicado en $1999^{1}$, la que proporcionó títulos de obras y fechas de composición. Dicha información fue contrastada y complementada con la revisión de partituras ${ }^{2}$, registros de inscripción (facilitados gentilmente por la Sociedad Chilena del Derecho de Autor), antecedentes recabados en la publicación biográfica realizada por Silvia León Smith ${ }^{3}$, y la información disponible en el sitio web oficial del compositor ${ }^{4}$.

La estructura en que se presenta la información toma como modelo de referencia el catálogo de la obra de León Schidlowsky llevado a cabo por David Schidlowsky y publicado en esta misma revista ${ }^{5}$. Se incluyen composiciones originales, pero sin excluir arreglos y orquestaciones cuando son de importancia artística para el compositor. Ante la extrema dificultad de recuperar fechas de estreno o primeras audiciones, se ha dado prioridad a la referencia de un registro fonográfico, que es el soporte en el que circula predominantemente la obra de Vicente Bianchi a

1 Torres, Rodrigo. 1999, "Bianchi, Vicente", Diccionario de la música española e hispanoamericana, vol. 2. Emilio Casares Rodicio (ed). Madrid: Sociedad General de Autores y Editores, pp. 432-434.

2 Agradecemos a Sergio Tapia Sandoval, transcriptor de partituras de Vicente Bianchi a formato digital, por facilitar el acceso a sus obras, además de indicar cuándo era pertinente la inclusión de un trabajo de arreglo u orquestación a la presente selección.

3 León Smith, Silvia, 2011. Vicente Bianchi. Músico por mandato divino. Santiago de Chile: Sociedad Chilena del Derecho de Autor (SCD).

4 www.vicentebianchi.scd.cl [Visitado el 14 de julio de 2017].

5 LXIX/224, julio-diciembre, 2015, pp. 37-72.

Revista Musical Chilena, Año LXXI, julio-diciembre, 2017, Nº 228, pp. 49-68 
lo largo del siglo XX. Se procuró agregar, junto con las referencias de discos de vinilo originales, reediciones en disco compacto ${ }^{6}$.

\section{A. LISTA DE OBRAS SEGÚN CLASIFICACIÓN POR GÉNEROS MUSICALES O CONFORMACIÓN INSTRUMENTAL, AÑO DE ESCRITURA Y NÚMERO EN EL CATÁLOGO CRONOLÓGICO}

\section{Canciones}

Amanecer

Rayito de sol

Despierta corazón

Esa noche

La ronda del beso

Redención

Tiernamente

Nuestra historia

No te vayas amor

Campito de mi tierra

Chile compañero

Jaraneo

Mi vals

Peruanita bonita

Tonadas de Manuel Rodríguez

A la bandera de Chile

Canto a Bernardo O'Higgins

Romance de los Carrera

Consejos por casamiento

Los finaos

Tiro y tiro, y el huaso firme

La refalaíta

Poema 15

Viña de mis amores

Un país llamado Chile

Primavera en tricolor

Santiago del Nuevo Extremo

Polvareda en el camino

Salitre

Elegía para cantar

La chilena

Antes de amarte

\section{Año}

1941

1941

1942

1942

1942

1942

1942

1952

1954

1954

1954

1954

1954

1954

1955

1956

1956

1956

1957

1957

1957

1957

1958

1960

1960

1960

1960

1962

1963

1963 (c.)

1964

1965

\section{Número del catálogo}

[O-4]

[O-5]

[O-8]

[O-9]

[O-10]

[O-11]

[O-12]

[O-18]

[O-26]

[O-27]

[O-28]

[O-29]

[O-30]

[O-31]

[O-32]

[O-34]

[O-35]

[O-36]

[O-40]

[O-41]

[O-42]

[O-43]

[O-44]

[O-49]

[O-51]

[O-54]

[O-55]

[O-56]

[O-57]

[O-58]

[O-61]

[O-69]

6 Hoy es posible acceder gratuitamente a digitalizaciones de buena calidad de la mayoría de los LP a los que se hace referencia en el catálogo. 


\section{Canciones}

No estés lejos de mí

Te amo y no te amo

No te quiero sino porque te quiero

Chile de mil caminos

Viene cabalgando el huaso

Epopeya triste

Estrofas a la Quintrala

En mayo pa' los 21

La balada del violín

Poema de aviso

Rancagua 1814

A Santa Teresa de Los Andes

La noche de Chillán

Cigarra de la tierra

La nerudiana

Farewell

Alma, no me digas nada

La independencia de Chile

\section{Comedias musicales}

Un misterio llamado mujer

La pérgola de las flores [F. Flores del Campo]

El mercado persa

\section{Coro mixto a capella}

Ave María

Chile fértil

\section{Coro y orquesta o conjunto}

La flor de la canela [M. I. Granda]

Tonadas de Manuel Rodríguez

Otra Navidad

A la bandera de Chile

Canto a Bernardo O'Higgins

Romance de los Carrera

Salitre

Guitarra de Nochebuena

La historia de Belén

Canción al niño Dios

Noche prodigiosa
Año

1965

1965

1965

1975

1975

1978

1978

1980

1984

1984

1984

1992

1997

2001

2004

2016

$\mathrm{s} / \mathrm{f}$

$\mathrm{s} / \mathrm{f}$

Año

1941

1960

1982-83

Año

1965

2016

Año

1953 (c.)

1955

1955

1956

1956

1956

1963

1964

1964

1964

1964
Número del catálogo

[O-70]

[O-71]

[O-72]

[O-84]

[O-85]

[O-88]

[O-89]

[O-91]

[O-101]

[O-102]

[O-103]

[O-108]

[O-111]

[O-113]

[O-114]

[O-116]

[O-118]

[O-120]

Número del catálogo

[O-6]

[O-50]

[O-98]

\section{Número del catálogo}

[O-68]

[O-117]

Número del catálogo

[O-24]

[O-32]

[O-33]

[O-34]

[O-35]

[O-36]

[O-57]

[O-60]

[O-62]

[O-63]

[O-64] 


\section{Coro y orquesta o conjunto}

Burrito de Belén

Refalando en Navidad

Misa a la Chilena

Misa del Huaso

Te Deum laudamus

Misa de la Cruz del Sur

Amén, Señor, amén

Oh Señor, envía tu espíritu

María de mi pueblo

Oración por la paz de Chile

En Belén, en Belén

Viene cabalgando el huaso

Epopeya triste

Estrofas a la Quintrala

María de Chile (letanías marianas)

Rancagua 1814

Totus tuo

Vamos pastores, vamos

Magnificat

\section{Himnos y marchas}

Himno del Club Deportivo U. Católica

Alegría de carnaval

Himno del Colegio Centellas

Himno del Comando de Industria e Ingeniería

Himno de SODIMAC

Himno del Colegio El Carmen Teresiano

Gloria a O'Higgins

Himno a los derechos humanos

Himno de la Universidad del Bí-Bío

Himno del Panathlon

Mar de Chile

Volvamos al estadio

Gloria en el mar

Himno de las Damas de la Defensa

Himno de la U. Tecnológica Metropolitana

Himno de la Dir. Gen. de Aeronáutica Civil

\section{Música para cine}

Amanecer

Rayito de sol

Un país llamado Chile

Escenas campesinas
Año

1964

1964

1964

1968

1969

1969

1969

1970

1973

1978

1974

1975

1978

1978

1984

1984

1986

1988

1989

Año

1942

1952

1970

1970

1975

1975

1978

1980

1980

1980

1980

1980

1982

1983

1994

$\mathrm{s} / \mathrm{f}$

Año

1941

1941

1960

1960

\section{Número del catálogo}

[O-65]

[O-66]

[O-67]

[O-74]

[O-75]

[O-76]

[O-77]

[O-78]

[O-81]

[O-82]

[O-83]

[O-85]

[O-88]

[O-89]

[O-100]

[O-103]

[O-104]

[O-105]

[O-106]

Número del catálogo

[O-13]

[O-17]

[O-79]

[O-80]

[O-86]

[O-87]

[O-90]

[O-92]

[O-93]

[O-94]

[O-95]

[O-96]

[O-97]

[O-99]

[O-110]

[O-119]

Número del catálogo

[O-4]

[O-5]

[O-51]

[O-52] 


\section{Orquesta}

Abejorros

Scherzando

Tonada rítmica

Tondero peruano [F. Ormeño]

Momento andino

El plebeyo [F. Pinglo]

Virgenes del sol [J. Bravo]

Carnaval arequipeño [tradicional]

Tristeza andina [C. Valderrama]

La palizada [A. Ayarza]

Marinera y tondero [E. López]

Cuándo y refalosa

Cantares chilenos

Danza de La Tirana [tradicional]

La refalaíta

La tranquera [O. Pérez]

Remolienda

Tríptico sinfónico

Los amores de una pollita

Escenas campesinas

Dos danzas de la Patria Vieja

Polvareda en el camino

Trote y cachimbo

La rosa y el clavel [J. Martínez]

Sajuria y sajuriana

Zamacuecas

A La Tirana

Rin y pericona

Piano

Preludio melancólico

Abejorros

Amanecer

Tonada rítmica

Variaciones en aire de cueca

Tonadas de Manuel Rodríguez

Canto a Bernardo O'Higgins

Romance de los Carrera

Sajuriando

Tradicionales de La Tirana

Bailando pericona

$\begin{array}{cc}\text { Año } & \text { Número del catálogo } \\ & \\ 1940 & {[\mathrm{O}-2]} \\ 1940 & {[\mathrm{O}-3]} \\ 1942 \text { (c.) } & {[\mathrm{O}-7]} \\ 1952 & {[\mathrm{O}-15]} \\ 1952 & {[\mathrm{O}-16]} \\ 1953 & {[\mathrm{O}-19]} \\ 1953 & {[\mathrm{O}-20]} \\ 1953(\text { c. }) & {[\mathrm{O}-21]} \\ 1953 & {[\mathrm{O}-22]} \\ 1953(\text { c. }) & {[\mathrm{O}-23]} \\ 1953(\text { c. } & {[\mathrm{O}-25]} \\ 1956 & {[\mathrm{O}-37]} \\ 1956 & {[\mathrm{O}-38]} \\ 1957(\text { c. }) & {[\mathrm{O}-39]} \\ 1957 & {[\mathrm{O}-43]} \\ 1958 & {[\mathrm{O}-45]} \\ 1958 & {[\mathrm{O}-46]} \\ 1958 & {[\mathrm{O}-47]} \\ 1959 & {[\mathrm{O}-48]} \\ 1960 & {[\mathrm{O}-52]} \\ 1960 \text { (c.) } & {[\mathrm{O}-53]} \\ 1962 & {[\mathrm{O}-56]} \\ 1964 & {[\mathrm{O}-59]} \\ 1967 & {[\mathrm{O}-73]} \\ 1991 & {[\mathrm{O}-107]} \\ 1993 & {[\mathrm{O}-109]} \\ 2009 & {[\mathrm{O}-115]} \\ \text { s } / \mathrm{f} & {[\mathrm{O}-121]} \\ & \end{array}$

\section{Año}

1932

1940

1941

1942 (c.)

1945

1955

1956

1956

2000

$\mathrm{s} / \mathrm{f}$

$\mathrm{s} / \mathrm{f}$

\section{Número del catálogo}

[O-1]

[O-2]

[O-4]

[O-7]

[O-14]

[O-32]

[O-35]

[O-36]

[O-112]

[O-122]

[O-123] 


\section{B. LISTADO CRONOLÓGICO DE OBRAS}

Para cada obra se señalan los siguientes antecedentes:

a) Título de la obra. Entre paréntesis, sus movimientos, cuando los tiene. Entre corchetes, nombre de compositor o procedencia del original, en el caso de orquestaciones o arreglos.

b) Año de composición.

c) Conformación instrumental (ver abreviaturas).

d) Duración aproximada (abreviado Dur).

e) Autor o procedencia del texto (abreviado Text).

f) Fonograma editado indicando tipo, título, intérpretes, lugar de edición, sello discográfico y año (Fon).

g) Editor, año de edición, cuando corresponda (abreviado $E d$ ).

i) Observaciones: fechas y lugares de estreno, de acuerdo con la información disponible, dedicatorias, premios, lugar de composición, etc. (abreviado Obs:).

\section{Abreviaturas}

$\begin{array}{llll}\text { A } & \text { contralto } & \text { cor } & \text { corno francés } \\ \text { ac } & \text { acompañamiento } & \text { cua } & \text { cuatro } \\ \text { arm } & \text { armonio } & \mathrm{cV} & \text { cuarteto vocal } \\ \text { arr } & \text { arreglo } & \mathrm{CD} & \text { disco compacto } \\ \text { arp } & \text { arpa } & \mathrm{DS} & \text { disco single } \\ \text { B } & \text { bajo (voz) } & \text { dir } & \text { director } \\ \text { b } & \text { bajo (a) } & \text { dir co } & \text { director de coro } \\ \text { Bar } & \text { barítono } & \text { dir inv } & \text { director invitado } \\ \text { bat } & \text { batería } & \text { dir orq } & \text { director de orquesta } \\ \text { bel } & \text { bells } & \text { fg } & \text { fagot } \\ \text { bom } & \text { bombo } & \text { fl } & \text { flauta } \\ \text { caj } & \text { caja } & \text { Fon } & \text { fonograma } \\ \text { Cass } & \text { cassette } & \text { glock } & \text { glockenspiel } \\ \text { cb } & \text { contrabajo } & \text { gui } & \text { guitarra } \\ \text { cdas } & \text { cuerdas } & \text { int } & \text { intérpretes } \\ \text { cha } & \text { charango } & \text { LP } & \text { Long Play } \\ \text { cl } & \text { clarinete } & \text { mand } & \text { mandolina } \\ \text { comp } & \text { fecha de composición } & \text { mar } & \text { maracas } \\ \text { comx } & \text { coro mixto } & \text { mari } & \text { marimba } \\ \text { coni } & \text { coro de niños } & \text { narr } & \text { narrador } \\ \text { conj } & \text { conjunto } & \text { ob } & \text { oboe }\end{array}$




$\begin{array}{llll}\text { org } & \text { órgano } & \text { tba } & \text { tuba } \\ \text { orq } & \text { orquesta } & \text { tbn } & \text { trombón } \\ \text { pand } & \text { pandero } & \text { timp } & \text { timbales } \\ \text { perc } & \text { percusión }(\mathrm{es}) & \text { tr } & \text { triángulo } \\ \text { pf } & \text { piano } & \text { tpt } & \text { trompeta } \\ \text { picc } & \text { piccolo } & \mathrm{V}, \mathrm{VV} & \text { voz, voces } \\ \text { pla } & \text { platillos } & \text { va } & \text { viola } \\ \mathrm{S} & \text { soprano } & \text { vibr } & \text { vibráfono } \\ \text { sol } & \text { solista }(\mathrm{s}) & \text { vlc } & \text { violonchelo } \\ \mathrm{T} & \text { tenor } & \text { vln } & \text { violín } \\ \text { tamb } & \text { tambor } & \text { xil } & \text { xilófono } \\ \text { tb } & \text { temple blocks } & & \end{array}$

[O-1] Preludio melancólico, 1932, pf, Dur. 5'40”, Fon: CD Vicente Bianchi con su piano a los 90, Vicente Bianchi (pf), Santiago: Autoedición, 2010, Ed. Vicente Bianchi con su piano a los 90, Santiago: Escuela Moderna de Música y Danza, Sociedad Chilena de Derecho de Autor SCD, 2011.

[O-2] Abejorros, 1940, orq: 2 fl, 2 ob, 2 cl, 2 fg, 2 cor, 3 tpt, 2 tbn, timp, perc, pf, vln I, vln II, vln III, vla, vlc, cb. Dur. 2'30”, Fon: LP Estampas Chilenas, Vicente Bianchi y su Gran Orquesta, Santiago de Chile: EMI-Odeón, 1964. CD 78 rpm Chile. Vicente Bianchi y su Orquesta. Santiago de Chile: Discos CNR, 2003, Ed. Transcripción digital de Celso Torres Mora, 2007. / pf, Fon: CD Vicente Bianchi con su piano a los 90, Vicente Bianchi (pf), Santiago: Autoedición, 2010, Ed. Vicente Bianchi con su piano a los 90, Santiago: Escuela Moderna de Música y Danza, Sociedad Chilena de Derecho de Autor SCD, 2011.

[O-3] Scherzando, quickstep, 1940, orq: 2 fl, 2 ob, 2 cl, 2 fg, 2 cor, 2 trp, 2 tbn, bat, vln I, vln II, vla, vlc, cb, Dur. 2'30”, Fon: CD 78 rpm Chile. Vicente Bianchi y su Orquesta. Santiago de Chile: Discos CNR, 2003, Ed. Transcripción digital de Sergio Tapia Sandoval, 2014.

[O-4] Amanecer, canción, 1941, Vy ac, Dur. 3'15”, Text. Alejandro Saa Silva. / orq, Fon: LP Éxitos chilenos de siempre, Vicente Bianchi y su Orquesta. Valentín Trujillo (pf). Santiago: EMI-Odeón, 1964. / V y orq, Fon: LP Si vas para Chile y otras canciones tradicionales, Vicente Bianchi y su Orquesta. Pedro Messone (V). Santiago: ARCI Music, 1969. / V y pf, Fon: CD Chile fértil. Vicente Bianchi (dir). Santiago de Chile: Autoedición, 2016, Ed. Santiago de Chile: Casa Amarilla, 1943. Clásicos de la Música Popular Chilena, Santiago: Ediciones Universidad Católica de Chile, 1994, pp. 97-100. / pf, Fon: CD Vicente Bianchi con su piano a los 90, Vicente Bianchi (pf), Santiago: Autoedición, 2010, Ed. Vicente Bianchi con su piano a los 90, Santiago: Escuela Moderna de Música y Danza, Sociedad Chilena de Derecho de Autor SCD, 
2011, Obs. Compuesta para la banda Sonora de la película Amanecer de esperanzas (1941), de Miguel Frank.

[O-5] Rayito de sol, vals, 1941, V y ac, Text. Alejandro Saa Silva, Obs. Compuesta para la banda Sonora de la película Amanecer de esperanzas (1941), de Miguel Frank.

[O-6] Un misterio llamado mujer, comedia musical, 1941, Text. Libreto de Carlos Cariola.

[O-7] Tonada rítmica, c.1942, orq: 2 fl, 2 ob, 2 cl, 2 fg, 2 cor, 2trp, tbn, tba, timp, perc, vln I, vln II, vla, vlc, cb, Dur. 3'30”, Ed. Transcripción digital de Sergio Tapia Sandoval. / pf, Fon: CD Vicente Bianchi con su piano a los 90, Vicente Bianchi (pf), Santiago: Autoedición, 2010, Ed. Vicente Bianchi con su piano a los 90, Santiago: Escuela Moderna de Música y Danza, Sociedad Chilena de Derecho de Autor SCD, 2011.

[O-8] Despierta corazón, bolero, 1942, V y ac, Dur. 3'10”, Text. Jorge Orellana, Fon: LP Los grandes éxitos de Lucho Gatica con Vicente Bianchi y su gran orquesta. Lucho Gatica (V), Vicente Bianchi (dir). Santiago de Chile: Odeón, c. 1957. CD 78 rpm Chile. Vicente Bianchi y su Orquesta. Santiago de Chile: Discos GNR, 2003.

[O-9] Esa noche, bolero, 1942, V y orq, Dur.3'30", Text. Jorge Orellana, Fon: LP Los grandes éxitos de Lucho Gatica con Vicente Bianchi y su gran orquesta. Lucho Gatica (V), Vicente Bianchi (dir). Santiago de Chile: Odeón, c. 1957. LP Boleros de todos los tiempos. Los Huasos Quincheros. Vicente Bianchi y su Orquesta. Santiago de Chile: London Records-Odeón Chile, 1965.

[O-10] La ronda del beso, 1942, V y ac, Text. Gustavo Martínez.

[O-11] Redención, 1942, V y ac, Text. Jorge Orellana, Fon: Cass Misceláneos. Los Quincheros. Santiago de Chile: EMI, 1993.

[O-12] Tiernamente, 1942, V y ac, Text. Jorge Orellana.

[O-13] Himno del Club Deportivo de la Universidad Católica, 1942, V, comx y orq, Dur. 2'20”, Text. Charles Bown Shirer, Fon: DS, 1943. Obs. Compuesto en base a dos marchas del siglo XIX, siendo una de ellas Tramp!, Tramp!, Tramp! (La esperanza del prisionero), de George Frederick Root (comp. 1864), tomada por Bianchi de un cancionero publicado por el Santiago College. Actualmente se emplea como himno oficial tanto del Club Deportivo como de la Pontificia Universidad Católica de Chile.

[O-14] Variaciones en aire de cueca, 1945, pf, Dur. 3'30", Fon: CD Vicente Bianchi con su piano a los 90, Vicente Bianchi (pf), Santiago: Autoedición, 2010, Ed. Vicente Bianchi con su piano a los 90, Escuela Moderna de Música y Danza, Sociedad Chilena de Derecho de Autor SCD, 2011.

[O-15] Tondero peruano, [original de Filomeno Ormeño Belmonte, Perú, 18991975], arr: 1952, orq: fl, ob, 2cl, fg, cor, 2 trp, tbn, timp, bat, pf, vln I, vln 
II, vla, vlc, cb. Dur. 2'50”, Fon: LP Recuerdo de Perú. Vicente Bianchi y su Orquesta. Trío los Jaranistas (VV). Santiago de Chile: Odeón, 1956, Ed. Transcripción digital de Celso Torres Mora, 2011, Obs. Compuesto durante la estadía del compositor en Lima, trabajando para Radio El Sol.

[O-16] Momento andino, 1952, orq: fl, vln I, vln II, vla, vlc, cb. Dur. 5', Fon: Grabado el 2/11/2016 en el Teatro Municipal de Ñunoa. Orquesta de Cámara de Chile. Alejandra Urrutia (dir). Inédito. Ed. Transcripción digital de Sergio Tapia Sandoval, 2008, Obs. Originalmente titulada Momento Incaico. Compuesta durante la estadía del compositor en Lima, trabajando para radio El Sol.

[O-17] Alegría de carnaval, marcha, 1952, Dur. 2', Text. Juan Rivero, Fon: DS Alegría de Carnaval. Orquesta Radio El Sol. E. Pizarro (V), Vicente Bianchi (dir). Lima: Sono Radio, 1953, Obs. Ganadora del segundo premio en el Carnaval Peruano de 1952.

[O-18] Nuestra historia, bolero, 1952, V y orq, Dur. 3', Text. Juan Rivero, Fon: LP Los grandes éxitos de Lucho Gatica con Vicente Bianchi y su gran orquesta. Lucho Gatica (V), Vicente Bianchi (dir). Santiago de Chile: Odeón, c. 1957.

[O-19] El plebeyo, vals peruano [original de Felipe Pinglo Alva, Perú, 1899-1936], arr: 1953, orq: fl, ob, 2 cl, fg, cor, 2 trp, tbn, bat, gui, pf, vln I, vln II, vln III, vla, vlc, cb, Dur. 2'40", Fon: LP Recuerdo de Perú. Vicente Bianchi y su Orquesta. Trío los Jaranistas (VV). Santiago de Chile: Odeón, 1956. CD Colección Inmortales: Vicente Bianchi, Vicente Bianchi y su Orquesta, Chile: EMI Music, 2004 [reed], Ed. Transcripción digital de Celso Torres Mora, 2011, Obs. Compuesto durante la estadía del compositor en Lima, trabajando para radio El Sol.

[O-20] Vírgenes del sol, fox incaico [original de Jorge Bravo de Rueda, Perú, 18951940], arr: 1953, orq: fl, ob, cl, fg, cor, 2 trp, tbn, timp, bat, pf, vln I, vln II, vln III, vla, vlc, cb, Dur. 2'40”, Fon: LP Recuerdo de Perú. Vicente Bianchi y su Orquesta. Trío los Jaranistas (VV). Santiago de Chile: Odeón, 1956. CD Colección Inmortales: Vicente Bianchi, Vicente Bianchi y su Orquesta, Chile: EMI Music, 2004 [reed], Ed. Transcripción digital de Celso Torres Mora, 2011.

[O-21] Carnaval arequipeño [tradicional], arr: c. 1953, orq: fl, ob, cl, fg, cor, 2 trp, tbn, bat, pf, vln I, vln II, vln III, vla, vlc, cb, Dur. 3', Fon: LP Recuerdo de Perú. Vicente Bianchi y su Orquesta. Trío los Jaranistas (VV). Santiago de Chile: Odeón, 1956, Ed. Transcripción digital Celso Torres Mora, 2011.

[O-22] Tristeza andina [original de Carlos Valderrama Herrera, Perú, 1887-1950], arr: 1953, pf y cdas: pf, vln I, vln II, vln III, vla, vlc, cb. Dur. 4'10”, Fon: LP Recuerdo de Perú. Vicente Bianchi y su Orquesta. Trío los Jaranistas (VV). Santiago de Chile: Odeón, 1956, Ed. Transcripción digital Celso Torres Mora, 2011. 
[O-23] La palizada, vals peruano [original de Alejandro Ayarza de Morales, Perú, 1884-1955], arr: c. 1953, orq: fl, ob, 2 cl, fg, cor, 2 trp, tbn, bat, gui, pf, vln I, vln II, vln III, vla, vlc, cb, Dur. 3'10", Fon: LP Recuerdo de Perú. Vicente Bianchi y su Orquesta. Trío los Jaranistas (VV). Santiago de Chile: Odeón, 1956. CD Colección Inmortales: Vicente Bianchi, Vicente Bianchi y su Orquesta, Chile: EMI Music, 2004 [reed], Ed. Transcripción digital Celso Torres Mora, 2011.

[O-24] La flor de la canela, vals peruano [original de María Isabel "Chabuca" Granda, Perú, 1920-1983], arr: c.1953, VV y orq: ST, 2 fl, ob, 2 cl, fg, cor, 2 trp, tbn, gui I, gui II, pf, vln I, vln II, vln III, vla, vlc, cb, Dur. 3'20”, Fon: LP Recuerdo de Perú. Vicente Bianchi y su Orquesta. Trío los Jaranistas (VV). Santiago de Chile: Odeón, 1956, Ed. Transcripción digital Celso Torres Mora, 2011.

[O-25] Marinera y tondero, [original de Ernesto López Mindreau, Perú, 18921972], arr: c. 1953, orq: fl, ob, 2 cl, fg, cor, 2 trp, tbn, timp, pla, tamb, bom, arp, vln I, vln II, vla, vlc, cb, Dur. 3'10”, Fon: CD Colección de Oro, volumen 1. Música peruana interpretada por la Orquesta Filarmónica de Lima. Miguel Harth-Bedoya (dir). Lima: Banco Latino, s/f, Ed. Transcripción digital Celso Torres Mora, 2011.

[O-26] No te vayas amor, bolero, 1954, V y orq, Dur. 2'20", Text. Félix Armando Núñez, Fon: LP Los grandes éxitos de Lucho Gatica con Vicente Bianchi y su gran orquesta. Lucho Gatica (V), Vicente Bianchi (dir). Santiago de Chile: Odeón, c. 1957. LP Ayúdeme usted compadre. De la banda de sonido del film de Germán Becker. Myriam (V), Vicente Bianchi (dir). Santiago de Chile: EMI-Odeón, 1968.

[O-27] Campito de mi tierra, tonada, 1954, VV y ac, Dur. 3'20”, Text. Juan Rivero, Fon: LP Rosita de Cachapoal. Silvia Infantas y Los Cóndores. Vicente Bianchi y su orquesta. Santiago de Chile: EMI-Odeón, 1964.

[O-28] Chile compañero, tonada, 1954, V y ac, Dur. 3'30", Text. Juan Rivero, Fon: LP Música para la Historia de Chile. Silvia Infantas y los Baqueanos. Vicente Bianchi y su Orquesta. Santiago de Chile, EMI-Odeón, 1959 [reed CD, Colección Bicentenario, Santiago de Chile: EMI, 2007].

[O-29] Jaraneo, 1954, V y ac, Text. Juan Rivero.

[O-30] Mi vals, vals peruano, 1954, V y ac, Dur. 2'50”, Text. Elena Toledo Crovetto, Fon: LP Recuerdo de Perú. Vicente Bianchi y su Orquesta. Trío los Jaranistas (VV). Santiago de Chile: Odeón, 1956, Obs. Compuesto durante la estadía del compositor en Lima, trabajando para radio El Sol.

[O-31] Peruanita bonita, vals peruano, 1954, V y ac, Dur. 2'40", Text. Juan Rivero, Fon: LP La Jarana es con... Lucila Campos (V). Perú: Virrey, 1974, Obs. Compuesto durante la estadía del compositor en Lima trabajando para radio El Sol. Ha alcanzado popularidad y múltiples grabaciones en Perú. 
[O-32] Tonadas de Manuel Rodríguez, tonada, 1955, V y ac, Dur. 5', Text. Pablo Neruda. / comx y orq: SATB, fl, ob, cl, 2 trp, pand, vln I, vln II, vln III, vla, vlc, cb, Fon: CD Neruda y Bianchi en canciones. Orquesta Clásica y Coro Polifónico de la Universidad de Santiago. Santiago Meza (dir orq), Santiago Marín (dir co), Vicente Bianchi (dir inv). Santiago de Chile: Oveja Negra, 2006, Ed. Transcripción digital de Sergio Tapia Sandoval, 2013. / VV y orq, Fon: LP Música para la Historia de Chile. Silvia Infantas y los Baqueanos. Vicente Bianchi y su Orquesta. Santiago: EMI-Odeón, 1959 [reed CD, Colección Bicentenario, Santiago de Chile: EMI, 2007]. / VV y conj, Fon: LP Esta tierra nuestra... Coro Chile Canta. Vicente Bianchi (dir). Santiago de Chile: EMI-Odeón, 1965. / V y pf, Ed. Santiago de Chile: Casa Amarilla, 1959. Clásicos de la Música Popular Chilena, Santiago: Ediciones Universidad Católica de Chile, 1994, pp. 101-104 / pf, Fon: CD Vicente Bianchi con su piano a los 90, Vicente Bianchi (pf), Santiago: Autoedición, 2010, Ed. Vicente Bianchi con su piano a los 90, Santiago: Escuela Moderna de Música y Danza, Sociedad Chilena de Derecho de Autor SCD, 2011.

[O-33] Otra Navidad, canción, 1955, comx y ac, Dur. 3', Text. Juan Rivero, Fon: LP Navidad en Chile. Coro Santa Marta. Vicente Bianchi (dir). Santiago de Chile: EMI-Odeón, 1965. LP Chile canta a su niño Dios. Coro Santa Marta. Vicente Bianchi (dir). Santiago de Chile: Ediciones Paulinas, 1973.

[O-34] A la bandera de Chile, 1956, V y ac, Dur. 3' 10", Text. Pablo Neruda. / V y conj: Fon: DS. Los Fortineros. Alejandra Bianchi y su conjunto. Santiago de Chile: IRT, 1973/ V y orq: Fon: CD Neruda y Bianchi en canciones. Orquesta Clásica y Coro Polifónico de la Universidad de Santiago. Santiago Meza (dir orq), Santiago Marín (dir co), Vicente Bianchi (dir inv). Santiago de Chile: Oveja Negra, 2006. Obs. Canción ganadora del segundo lugar en la competencia folklórica del XIV Festival Internacional de la Canción de Viña del Mar, 1973. Los Fortineros (int).

[O-35] Canto a Bernardo O'Higgins, canción, 1956, V y ac, Dur. 4' 10”, Text. Pablo Neruda. / V y orq: 2 fl, 2 ob, 2 cl, 2 fg, 2 cor, 2 trp, timp, perc, vln I, vln II, vla, vlc, cb, Fon: CD Neruda y Bianchi en canciones. Orquesta Clásica y Coro Polifónico de la Universidad de Santiago. Santiago Meza (dir orq), Santiago Marín (dir co), Vicente Bianchi (dir inv). Santiago de Chile: Oveja Negra, 2006, Ed. Transcripción digital de Sergio Tapia Sandoval, 2010. / VV y orq, Fon: LP Música para la Historia de Chile. Silvia Infantas y los Baqueanos. Vicente Bianchi y su Orquesta. Santiago: EMI-Odeón, 1959 [reed CD, Colección Bicentenario, Santiago de Chile: EMI, 2007]. / V y pf, $E d$. Santiago de Chile: Southern Music International, 1961. / pf, Fon: CD Vicente Bianchi con su piano a los 90, Vicente Bianchi (pf), Santiago: Autoedición, 2010, Ed. Vicente Bianchi con su piano a los 90, Santiago: Escuela Moderna de Música y Danza, Sociedad Chilena de Derecho de Autor SCD, 2011. 
[O-36] Romance de los Carrera, canción, 1956, V y ac, Dur. 5', Text. Pablo Neruda. / V y orq: 2 fl, 2 ob, 2 cl, 2 fg, 2 cor, 2 trp, vln I, vln II, vla, vlc, cb, Fon: LP Ayúdeme usted compadre. De la banda de sonido del film de Germán Becker. Myriam (V), Vicente Bianchi (dir). Santiago de Chile: EMI-Odeón, 1968. CD Neruda y Bianchi en canciones. Orquesta Clásica y Coro Polifónico de la Universidad de Santiago. Santiago Meza (dir orq), Santiago Marín (dir co), Vicente Bianchi (dir inv). Santiago de Chile: Oveja Negra, 2006, Ed. Transcripción digital de Sergio Tapia Sandoval, 2004. / VV y orq, Fon: LP Música para la Historia de Chile. Silvia Infantas y los Baqueanos. Vicente Bianchi y su Orquesta. Santiago: EMI-Odeón, 1959 [reed CD, Colección Bicentenario, Santiago de Chile: EMI, 2007]. / VV y conj, Fon: LP Chile en una tonada. Los Huasos Quincheros. Santiago: EMI-Odeón, 1967. / V y pf, Ed. Santiago de Chile: Casa Amarilla, 1959. / pf, Fon: CD Vicente Bianchi con su piano a los 90, Vicente Bianchi (pf), Santiago: Autoedición, 2010, Ed. Vicente Bianchi con su piano a los 90, Santiago: Escuela Moderna de Música y Danza, Sociedad Chilena de Derecho de Autor SCD, 2011.

[O-37] Cuando y refalosa (I: Cuando, II: Refalosa), 1956, orq: picc, 2 fl, 2 ob, 2 cl, 2 fg, 4 cor, 3 trp, 2 tbn, arp, glock, caj, timp, vln I, vln II, vla, vlc, cb. Dur.5'30”, $E d$. Transcripción digital de Sergio Tapia Sandoval.

[O-38] Cantares chilenos, 1956, orq: fl, ob, 2 cl, fg, 2 trp, tbn, timp, bat, pf, vln I, vln II, vla, vlc, cb. Dur. 4'30”, Fon: LP Cantares chilenos, Vicente Bianchi y su Orquesta, Santiago de Chile: EMI-Odeón, 1969, Ed. Transcripción digital de Sergio Tapia Sandoval, Obs. Fantasía orquestal sobre El copihue rojo (Ignacio Verdugo), Río, río (tradicional), Ay, ay, ay (Osmán Pérez Freire), y 25 limones (tradicional).

[O-39] Danza de La Tirana, danza nortina [tradicional], Arr: c.1957, orq, Dur.2', Fon: LP Estampas Chilenas, Vicente Bianchi y su Gran Orquesta, Santiago de Chile: EMI-Odeón, 1964. CD Colección Inmortales: Vicente Bianchi, Vicente Bianchi y su Orquesta, Chile: EMI Music, 2004 [reed].

[O-40] Consejos por casamiento, cuando, 1957, V y ac, Dur. 3'20", Text. Hernán Arenas, Fon: LP Música para la Historia de Chile. Silvia Infantas y los Baqueanos. Vicente Bianchi y su Orquesta. Santiago de Chile, EMI-Odeón, 1959 [reed CD, Colección Bicentenario, Santiago de Chile: EMI, 2007]. Obs. Basada en El cuando (tradicional).

[O-41] Los finaos, cueca, 1957, V y ac, Text. Hernán Arenas, Fon: LP Fiesta chilena, vol. 4. Silvia Infantas y los Baqueanos (int). Vicente Bianchi y su Orquesta (int). Santiago de Chile: Odeón, 1959.

[O-42] Tiro y tiro, y el huaso firme, tonada, 1957, V y ac, Dur. 3'15”, Text. Hernán Arenas, Fon: LP Rosita de Cachapoal. Silvia Infantas y Los Cóndores. Vicente Bianchi y su orquesta. Santiago de Chile: EMI-Odeón, 1964.

[O-43] La refalaíta, refalosa, 1957, V y ac, Dur. 2'30”, Text. Hernán Arenas, Fon: LP Música para la Historia de Chile. Silvia Infantas y los Baqueanos. Vicente 
Bianchi y su Orquesta. Santiago de Chile, EMI-Odeón, 1959 [reed CD, Colección Bicentenario, Santiago de Chile: EMI, 2007]. / 1958, orq: Dur. 1'55", Fon: LP Estampas Chilenas, Vicente Bianchi y su Gran Orquesta, Santiago de Chile: EMI-Odeón, 1964. CD Vicente Bianchi, creador y maestro. Vicente Bianchi y su Orquesta. EMI-Odeón, 1997 [reed].

[O-44] Poema 15, 1958, V y ac, Dur. 2'40", Text. Pablo Neruda, Fon: LP Boleros de todos los tiempos. Los Huasos Quincheros. Vicente Bianchi y su Orquesta. Santiago de Chile: London Records-Odeón Chile, 1965. CD Neruda y Bianchi en canciones. Orquesta Clásica y Coro Polifónico de la Universidad de Santiago. Santiago Meza (dir orq), Santiago Marín (dir co), Vicente Bianchi (dir inv). Santiago de Chile: Oveja Negra, 2006.

[O-45] La tranquera, [original de Osmán Pérez Freire, Chile, 1878-1930], arr: 1958, pf y cdas: pf, vln I, vln II, vla, vlc, cb, Dur. 3', Fon: LP Cantares chilenos, Vicente Bianchi y su Orquesta, Santiago de Chile: EMI-Odeón, 1969. CD Colección Inmortales: Vicente Bianchi, Vicente Bianchi y su Orquesta, Chile: EMI Music, 2004 [reed], Ed. Transcripción digital de Sergio Tapia Sandoval, 2008.

[O-46] Remolienda, cueca, 1958, orq, Dur: 3', Fon: LP Estampas Chilenas, Vicente Bianchi y su Gran Orquesta, Santiago de Chile: EMI-Odeón, 1964.

[O-47] Tríptico sinfónico (I: Tonada de Manuel Rodríguez, II: Romance de los Carrera, III: Canto a O'Higgins) , 1958, orq: fl picc, $2 \mathrm{fl}, 2$ ob, 2 cl, 2 fg, 4 cor, 3 trp, 3 tbn, tu, timp, glock, perc, pf (arp), vln I, vln II, vla, vlc, cb, Dur. 6', Fon: LP Estampas Chilenas, Vicente Bianchi y su Gran Orquesta, Santiago de Chile: EMI-Odeón, 1964. CD Colección Inmortales: Vicente Bianchi, creador y maestro. Vicente Bianchi y su Orquesta. EMI-Odeón, 1997 [reed], Ed. Transcripción digital de Sergio Tapia Sandoval.

[O-48] Los amores de una pollita, 1959, orq: picc, 2 fl, 2 ob, 2 cl, 2 fg, 4 cor, 3 trp, 3 tbn, tba, timp, xil, perc, vln I, vln II, vla, vlc, cb, Dur. 2'40", Fon: LP Estampas Chilenas, Vicente Bianchi y su Gran Orquesta, Santiago de Chile: EMI-Odeón, 1964, Ed. Transcripción digital de Sergio Tapia Sandoval, Obs. Basada en la tonada La pollita (tradicional).

[O-49] Viña de mis amores, canción, 1960, V y ac, Dur. 2'30", Text. Mario Gaymer, Fon: LP Sonia y Myriam en La Habana. Sonia y Myriam (VV). La Habana: Kubaney, 1960, Ed. V y pf: Santiago de Chile: Casa Amarilla, 1961, Obs. Canción ganadora del segundo lugar (oficialmente "mención honrosa") en la primera versión del Festival de la Canción de Viña del Mar, 1960.

[O-50] La pérgola de las flores, comedia musical [música de Francisco Flores del Campo, texto de Isidora Aguirre], Arr: 1960, VV y conj: pf I, pf II, bat, cb. Fon: LP La Pérgola de las Flores. Santiago de Chile: Philips, 1960. CD Santiago de Chile: Star Sound, 1996 [reed]. Obs. Manuscrito con fecha de marzo de 1960. Transcripción a notación musical y orquestación de las canciones originales de Flores del Campo. 
[O-51] Un país llamado Chile, 1960, V y ac, Text. Marina Lara. Obs. Compuesta para el documental Un país llamado Chile (1961) de Boris Hardy.

[O-52] Escenas campesinas, 1960, orq, Dur. 4', Fon: LP Estampas Chilenas, Vicente Bianchi y su Gran Orquesta, Santiago de Chile: EMI-Odeón, 1964. CD Colección Inmortales: Vicente Bianchi, Vicente Bianchi y su Orquesta, Chile: EMI Music, 2004 [reed], Obs. Música incidental para el documental Un país llamado Chile (1961) de Boris Hardy.

[O-53] Dos danzas de la Patria Vieja (I:El Aire, II: El Pequén), c.1960, orq: fl, ob, 3 cl, tbn, bat, gui, arp, pf, vln I, vln II, vln III, vla, vlc, cb. Dur. 3', Fon: LP Estampas Chilenas, Vicente Bianchi y su Gran Orquesta, Santiago de Chile: EMI-Odeón, 1964. CD Vicente Bianchi, creador y maestro. Vicente Bianchi y su Orquesta. EMI-Odeón, 1997 [reed], Ed. Transcripción digital de Sergio Tapia Sandoval.

[O-54] Primavera en tricolor, 1960, V y ac, Text. Marina Lara.

[O-55] Santiago del Nuevo Extremo, 1960, V, comx y orq, Dur. 3'20", Text. Marina Lara, Fon: CD Chile fértil. Vicente Bianchi (dir). Santiago de Chile: Autoedición, 2016.

[O-56] Polvareda en el camino (Adiós a los Cuatro Huasos), 1962, V y ac, Dur. 3'30", Text. Diego Barros de Ortiz, Fon: LP De mañanita... Los Quincheros. Santiago de Chile: EMI-Odeón, 1964. / orq: 2 fl, 2 ob, 2 cl, 2 fg, 2 cor, 2 trp, 2 tbn, timp, bat, arp, pf, vln I, vln II, vla, vlc, cb, Dur. 3'30”, Fon: LP Estampas Chilenas, Vicente Bianchi y su Gran Orquesta, Santiago de Chile: EMI-Odeón, 1964, CD Colección Inmortales: Vicente Bianchi, Vicente Bianchi y su Orquesta, Chile: EMI Music, 2004 [reed], Ed. Transcripción digital de Sergio Tapia Sandoval.

[O-57] Salitre, cachimbo, 1963, comx y orq, Dur. 4'10", Text. Pablo Neruda, Fon: CD Neruda y Bianchi en canciones. Orquesta Clásica y Coro Polifónico de la Universidad de Santiago. Santiago Meza (dir orq), Santiago Marín (dir co), Vicente Bianchi (dir inv). Santiago de Chile: Oveja Negra, 2006.

[O-58] Elegía para cantar, c. 1963, V y ac, Dur. 5’30”, Text. Pablo Neruda, Fon: CD Neruda y Bianchi en canciones. Orquesta Clásica y Coro Polifónico de la Universidad de Santiago. Santiago Meza (dir orq), Santiago Marín (dir co), Vicente Bianchi (dir inv). Santiago de Chile: Oveja Negra, 2006.

[O-59] Trote y cachimbo, 1964, orq: picc, 2 fl, 2 ob, 2 cl, 2 fg, 4 cor, 2 trp, 3 tbn, tba, timp, glock, perc I, perc II, vln I, vln II, vla, vlc, cb, Dur. 5'20”, Ed. Transcripción digital de Sergio Tapia Sandoval, 2008.

[O-60] Guitarra de Nochebuena, villancico, 1964, V, comx y ac, Dur. 2'40", Text. Germán Becker Ureta, Fon: LP Misa a la chilen a y otros 6 temas chilenos. Coro Chile Canta y Orquesta. Jaime Soval (V), Vicente Bianchi (dir), Santiago de Chile: EMI-Odeón, 1965. LP Navidad en Chile. Coro Santa Marta. Vicente Bianchi (dir). Santiago de Chile: EMI-Odeón, 1965. 
[O-61] La chilena, tonada, 1964, V y ac, Dur. 2'10”, Text. Germán Becker Ureta, Fon: LP Ayúdeme usted compadre. De la banda de sonido del film de Germán Becker. Arturo Gatica (V), Vicente Bianchi (dir). Santiago de Chile: EMI-Odeón, 1968.

[O-62] La historia de Belén, oratorio de Navidad (I: Prólogo, II: Elogio al trabajo, III: La anunciación, IV: Magnificat, V: Ronda angelical a Maria, VI: Encuentro del Ángel con José, VII: Lectura del edicto, VIII: La peregrinación, IX: Noche prodigiosa, X: Canción de cuna, XI: Vamos, pastores, vamos, XII: Los reyes magos, XIII: Aleluy a al Señor, ), 1964, narr, sols, conj, comx y coni, Dur. 50', Text. Vicente Bianchi. Obs. Estrenado en 1970, y posteriormente revisado y reestrenado en 1988 en la Quinta Vergara, de Viña del Mar. Algunas piezas circularon de forma independiente, ver O-63, O-64 y O-105.

[O-63] Noche prodigiosa, canción, 1964, comx y ac, Dur. 2'20”, Text. Vicente Bianchi, Fon: LP Chile canta a su niño Dios. Coro Santa Marta. Vicente Bianchi (dir). Santiago de Chile: Ediciones Paulinas, 1973, Obs. Pieza que forma parte del oratorio de Navidad La Historia de Belén (O-62) (movimiento IX).

[O-64] Canción de cuna al niño Dios, 1964, comx y ac, Dur. 2', Text. Vicente Bianchi, Fon: LP Chile canta a su niño Dios. Coro Santa Marta. Vicente Bianchi (dir). Santiago de Chile: Ediciones Paulinas, 1973, Obs. Pieza que forma parte del oratorio de Navidad La Historia de Belén (O-62), con título Canción de cuna (movimiento X).

[O-65] Burrito de Belén, trote-villancico, 1964, comx y conj, Dur. 2'50”, Text. Germán Becker Ureta, Fon: LP Misa a la chilena y otros 6 temas chilenos. Coro Chile Canta y Orquesta. Jaime Soval (V), Vicente Bianchi (dir), Santiago de Chile: EMI-Odeón, 1965. LP Navidad en Chile. Coro Santa Marta. Vicente Bianchi (dir). Santiago de Chile: EMI-Odeón, 1965. LP Chile canta a su niño Dios. Coro Santa Marta. Vicente Bianchi (dir). Santiago de Chile: Ediciones Paulinas, 1973. / 1987, conj y comx: SATB, pand, pf, cb, Ed. Transcripción digital de Sergio Tapia Sandoval, 2014.

[O-66] Refalando en Navidad, refalosa, 1964, comx y ac, Text. Vicente Bianchi.

[O-67] Misa a la Chilena (I: Introito, II: Kyrie, III: Gloria, IV: Credo, V: Ofertorio, VI: Santo, VII: Padre nuestro, VIII: Cordero de Dios, IX: María de mi pueblo, X: Canto de salida), 1964-1965, orq. y comx: SATB, bat, pand, bom, gui, cha, pf, vlc, cb, Dur. 21', Text. Rubén Campos (mov. IX). Textos litúrgicos del Missale Romanum. Traducidos con licencia arzobispal. Fon: LP Misa a la Chilena y otros 6 temas chilenos. Coro Chile Canta y Orquesta. Jaime Soval (V), Vicente Bianchi (dir), Santiago de Chile: EMI-Odeón, 1965 (solo movs. II, III, IV, VI, VIII y X), LP Voces del cobre, Coro del Mineral El Teniente, Rosenda Vega (S), Guillermo Contreras (T), Eric Cuevas (Bar), Nolberto González Hevia (dir), Santiago de Chile: Polydor, 1973. CD Vicente Bianchi, creadory maestro. Vicente Bianchi y su Orquesta. EMI-Odeón, 1997 [reed] (solo movs. II, III, IV, VI, VIII y X), Ed. Transcripción digital de Sergio Tapia Sandoval, 2014, 
Obs. Estrenada a fines de mayo de 1965 en la inauguración de la parroquia Santa Adela de Cerrillos, presidida por el cardenal Raúl Silva Henríquez. Los movimientos I, V, VII corresponden a la Misa del Huaso (O-74), y el movimiento IX a la composición de Bianchi para su hija Bernardita (O80), siendo agregados de forma posterior a la versión de 1965. Manuscritos para $\mathbf{V}$ y pf, comx y conj, y comx donados por el compositor a la Biblioteca Nacional.

[O-68] Ave María, 1965, comx: SATB, Dur. 2'10", Text. Traducción tradicional del texto latino. Obs. Partitura manuscrita con fecha octubre de 1965. Compuesto con motivo de la Primera Comunión de Silvana Bianchi, hija del compositor.

[O-69] Antes de amarte, 1965, Vy ac, Dur. 3'30", Text. Pablo Neruda, Fon: CD Neruda y Bianchi en canciones. Orquesta Clásica y Coro Polifónico de la Universidad de Santiago. Santiago Meza (dir orq), Santiago Marín (dir co), Vicente Bianchi (dir inv). Santiago de Chile: Oveja Negra, 2006.

[O-70] No estés lejos de mí, 1965, V y ac, Dur. 2'40”, Text. Pablo Neruda, Fon: CD Neruda y Bianchi en canciones. Orquesta Clásica y Coro Polifónico de la Universidad de Santiago. Santiago Meza (dir orq), Santiago Marín (dir co), Vicente Bianchi (dir inv). Santiago de Chile: Oveja Negra, 2006.

[O-71] Te amo y no te amo, 1965, V y ac, Dur. 3'40", Text. Pablo Neruda, Fon: CD Neruda y Bianchi en canciones. Orquesta Clásica y Coro Polifónico de la Universidad de Santiago. Santiago Meza (dir orq), Santiago Marín (dir co), Vicente Bianchi (dir inv). Santiago de Chile: Oveja Negra, 2006.

[O-72] No te quiero sino porque te quiero, 1965, V y ac, Dur. 2'50", Text. Pablo Neruda, Fon: CD Neruda y Bianchi en canciones. Orquesta Clásica y Coro Polifónico de la Universidad de Santiago. Santiago Meza (dir orq), Santiago Marín (dir co), Vicente Bianchi (dir inv). Santiago de Chile: Oveja Negra, 2006.

[O-73] La rosa y el clavel, cueca [original de Jorge Martínez Serrano, comp. 1962], arr: 1967, orq: 2 fl, 2 ob, 2 cl, 2 fg, 4 cor, 2 trp, timp, glock, perc I, perc II, vln I, vln II, vla, vlc, cb, Dur. 3', Fon: LP Cantares chilenos, Vicente Bianchi y su Orquesta, Santiago de Chile: EMI-Odeón, 1969. CD Colección Inmortales: Vicente Bianchi, Vicente Bianchi y su Orquesta, Chile: EMI Music, 2004 [reed], Ed. Transcripción digital de Celso Torres Mora, 2009.

[O-74] Misa del Huaso (Misa comunitaria chilena) (I: Introito, II: Kyrie, III: Gloria, IV: Credo, V: Ofertorio, VI: Santo, VII: Padre nuestro, VIII: Cordero de Dios), 1967, comx, arm y conj, Dur. 18', Obs. Movimientos I, V y VII posteriormente agregados como cantos del propio en la Misa a la Chilena (O-67, movimientos I, V y VII).

[O-75] Te Deum laudamus, 1969, orq. y comx: SATB, 2 fl, 2 ob, 2cl, 2 trp, timp, vln I, vln II, vla, vlc, cb, Dur. 4’20”, Text: Pbro. Felipe Lázaro Urrizola, traducción del himno latino con licencia arzobispal, junio de 1969, Ed. Transcripción 
digital Sergio Tapia Sandoval, 2007, Obs. Encargado por el cardenal Raúl Silva Herníquez. Se interpretó cada 18 de septiembre en la Catedral de Santiago desde 1969 a 2000. Manuscrito para org, y comx y org donado por el compositor a la Biblioteca Nacional.

[O-76] Misa de la Cruz del Sur (Misa sudamericana) (I: Canto de entrada [Introito], II: Señor, ten piedad [Kyrie], III: Gloria, IV: Credo, V: Canto de las ofrendas [Ofertorio], VI: Santo [Sanctus], VII: Padre nuestro, VIII: Cordero de Dios [Agnus Dei], IX: Canto de paz y comunión, X: Canto de salida [Cueca de América]), 1969, orq. y comx: SATB, fl, perc, cha, cua, gui, org, pf, cb. Dur. 28', Text: Rubén Campos (movs. I, V, IX, X). Textos litúrgicos del Missale Romanum. Traducidos con licencia arzobispal. Fon: LP Misa de la Cruz del Sur, Misa Sudamericana. Cuarteto doble Americano y Orquesta. Vicente Bianchi (director). Santiago de Chile, EMI-Odeón, 1972. CD Vicente Bianchi, creadory maestro. Vicente Bianchi y su Orquesta. EMI-Odeón, 1997 [reed], Ed. Musikforlag, 1993.

[O-77] Amén, Señor, amén, 1969, co y ac, Dur. 2'50", Text. Vicente Bianchi.

[O-78] Oh Señor, envía tu Espiritu, 1970, orq. y comx: SATB, 2 fl, 2 ob, 2 cl, 2 fg, 2 cor, 2 trp, 2 tbn, timp, vln I, vln II, vla, vlc, cb. Dur. 2'40", Text. Versión en castellano de antífona extraída del Salmo 103 y de la secuencia Veni Creator Spiritus. Ed. Transcripción digital de Sergio Tapia Sandoval, 2015, Obs. Compuesta para la transmisión del mando al presidente de Chile Salvador Allende. Estrenada el 3 de noviembre de 1970 en el primer Te Deum ecuménico, en la Catedral de Santiago.

[O-79] Himno del Colegio Centellas, 1970, Text. Manuel Saavedra.

[O-80] Himno del Comando de Industria e Ingeniería, 1970, Text. Rodolfo Mayer.

[O-81] María de mi pueblo, 1970, comx y conj: SATB, gui, org, cb. Dur. 1'50", Text. Rubén Campos, Fon: LP Voces del cobre, Coro del Mineral El Teniente, Rosenda Vega (S), Guillermo Contreras (T), Eric Cuevas (Bar), Nolberto González Hevia (dir), Santiago de Chile: Polydor, 1973, Ed. Transcripción digital de Sergio Tapia Sandoval, 2014, Obs. Compuesta con motivo de la Primera Comunión Bernardita Bianchi, hija del compositor. Agregada a la Misa a la Chilena (O-67, movimiento IX).

[O-82] Oración por la paz de Chile, 1974, comx y orq: SATB, fl, ob, cl, 2 trp, timp, vln I, vln II, vla, vlc, cb. Dur. 1'20", Text. Mons. Raúl Silva Henríquez, Ed. Transcripción digital de Sergio Tapia Sandoval, 2015, Obs: También titulada Canto de paz. Estrenada en la Catedral de Santiago el 18 de septiembre de 1978, en la ceremonia del Te Deum Ecuménico de Fiestas Patrias.

[O-83] En Belén, en Belén, villancico, 1974, comx y conj: SATB, pf, bat, cb, Dur. 2'30", Text. Vicente Bianchi, Ed. Transcripción digital de Sergio Tapia Sandoval, 2014. 
[O-84] Chile de mil caminos, 1975, Text. Diego Barros Ortiz, Obs. Interpretada en vivo en el Teatro Municipal de Viña del Mar, 21 de septiembre de 2008. Los Huasos Quincheros (int).

[O-85] Viene cabalgando el huaso, 1975, VV y orq: 2 fl, 2 ob, 2 cl, 2 fg, 2 cor, 2 trp, 2 tbn, bat, timp, gui, vln I, vln II, vla, vlc, cb. Dur. 3', Text. Diego Barros Ortiz, Fon: LP Chile: canciones de hoy. Miguel Zabaleta (dir orq). Vicente Bianchi y su Coro. Santiago de Chile: Ministerio de Relaciones Exteriores de Chile, Dirección de Asuntos Culturales e Información Exterior (DINEX), 1984, Ed. Transcripción digital de Sergio Tapia Sandoval, Obs. Tonada ofrecida por el compositor al Club del Rodeo Chileno "Gil Letelier".

[O-86] Himno de SODIMAC, 1975, Text. Manuel Romero.

[O-87] Himno del Colegio El Carmen Teresiano, 1975, Text. Cecilia Cabrera.

[O-88] Epopeya triste, 1978, comx y orq, Dur. 3'20", Text. Héctor Rodríguez, Fon: CD Chile fértil. Vicente Bianchi (dir). Santiago de Chile: Autoedición, 2016.

[O-89] Estrofas a la Quintrala, 1978, V, comx y orq, Dur. 3'20", Text. Héctor Rodríguez, Fon: CD Chile fértil. Vicente Bianchi (dir). Santiago de Chile: Autoedición, 2016.

[O-90] Gloria a O'Higgins, marcha, 1978, Text. Julio Barrenechea, Obs. Compuesta para la celebración del natalicio de Bernardo O'Higgins Riquelme (17781842), interpretándose por única vez. Reestreno en la ciudad de Chillán, 2007. Banda Instrumental RI-9 Chillán. Nelson Rozas (dir).

[O-91] En mayo pa'los 21, 1980, Text. Germán Becker Ureta.

[O-92] Himno a los derechos humanos, 1980, Text. Manuel Bianchi.

[O-93] Himno de la Universidad del Bío-Bío, 1980, Text. Germán Becker Ureta.

[O-94] Himno del Panathlon, 1980, Text. Manuel Bianchi.

[O-95] Mar de Chile, marcha, 1980, Text. Manuel Bianchi.

[O-96] Volvamos al estadio, marcha, 1980, Text. Germán Becker Ureta.

[O-97] Gloria en el mar, marcha, 1982, Text. Germán Becker Ureta.

[O-98] El mercado persa, comedia musical, 1982-83, Text. Libreto de Rafael Sousa. Obs. La obra no pudo estrenarse.

[O-99] Himno de las Damas de la Defensa, 1983, Text. Nina Donoso.

[O-100] María de Chile (letanías marianas), 1984, VV y ac, Dur. 3', Text. Vicente Bianchi.

[O-101] La balada del violín, 1984, Text. Víctor Domingo Silva.

[O-102] Poema de aviso, 1984, Text. Orlando Cabrera Leiva. 
[O-103] Rancagua 1814, 1984, V, comx y orq, Dur. 3', Text. Humberto Tassara, Fon: CD Chile fértil. Vicente Bianchi (dir). Santiago de Chile: Autoedición, 2016.

[O-104] Totus tuo, 1986, comx y ac: SATB, Dur. 3'30”, Text. Vicente Bianchi.

[O-105] Vamos pastores, vamos, 1987, comx y conj: SATB, pand, pfm, cb, Dur. 2'30”, Text. Vicente Bianchi, Ed. Transcripción digital de Sergio Tapia Sandoval, 2016, Obs. Pieza que forma parte del oratorio de Navidad La Historia de Belén (O-62, movimiento XI). Interpretada el 21 de diciembre de 2011 en la Sala América, Biblioteca Nacional. Vicente Bianchi y su Ensamble Vocal (int).

[O-106] Magnificat, 1989, comx y orq, Dur. 3', Text. Traducción libre sin firma del texto litúrgico latino. Obs. Distinta a la composición del mismo título en O-62. Partitura manuscrita para coro y reducción al piano de la orquesta con fecha agosto de 1989.

[O-107] Sajuria y sajuriana, 1991, orq: 2 fl, 2 ob, 2 cl, 2 fg, 2 cor, 2 trp, 2 tbn, timp, caj, tb, glock, timp, vln I, vln II, vla, vlc, cb. Fon: Grabado el 2/11/2016 en el Teatro Municipal de Ñunoa. Orquesta de Cámara de Chile. Alejandra Urrutia (dir). Inédito. Ed. Transcripción digital de Sergio Tapia Sandoval, Obs. Fantasía sobre temas folklóricos.

[O-108] A Santa Teresa de Los Andes, 1992, V y ac, Dur. 3'30", Text. Pbro. Demetrio Bravo Santibáñez.

[O-109] Zamacuecas, 1993, orq: picc, 2 fl, 2 ob, 2 cl, 2 fg, 4 cor, 2 trp, 3 tbn, tba, timp, glock, perc I, perc II, vln I, vln II, vla, vlc, cb. Dur. 3'10”, Ed. Transcripción digital de Sergio Tapia Sandoval, 2016, Obs. Melodías recopiladas del folklore de la década de 1890.

[O-110] Himno de la Universidad Tecnológica Metropolitana, 1994, Text. Rubén Campos Aragón, Obs. Estrenado en 1994 en el salón del ex-Congreso Nacional, con motivo de la celebración del primer aniversario de la institución.

[O-111] La noche de Chillán, vals, 1997, V y ac, Dur. 3'20”, Text. Pablo Neruda, Fon: CD Neruda y Bianchi en canciones. Orquesta Clásica y Coro Polifónico de la Universidad de Santiago. Santiago Meza (dir orq), Santiago Marín (dir co), Vicente Bianchi (dir inv). Santiago de Chile: Oveja Negra, 2006. CD Chile fértil. Vicente Bianchi (dir). Santiago de Chile: Autoedición, 2016, Obs. Canción ganadora de la competencia folklórica del XXXIX Festival Internacional de la Canción de Viña del Mar, 1998. Santiago Cuatro (int). Manuscrito para pf donado por el compositor a la Biblioteca Nacional.

[O-112] Sajuriando, 2000, pf, Dur. 3'20”, Fon: CD Vicente Bianchi con su piano a los 90, Vicente Bianchi (pf), Santiago: Autoedición, 2010, Ed. Vicente Bianchi con su piano a los 90, Santiago: Escuela Moderna de Música y Danza, Sociedad Chilena de Derecho de Autor SCD, 2011. 
[O-113] Cigarra de la tierra, 2001, V y ac, Dur. 4', Text. Pepe Cornejo, Fon: CD Chile fértil. Vicente Bianchi (dir). Santiago de Chile: Autoedición, 2016. Obs. Canción clasificada en la competencia folklórica del XLIII Festival Internacional de la Canción de Viña del Mar, 2002.

[O-114] La nerudiana, cueca, 2004, V y ac, Dur. 1'30”, Text. José Cornejo Aliaga, Fon: CD Neruda y Bianchi en canciones. Orquesta Clásica y Coro Polifónico de la Universidad de Santiago. Santiago Meza (dir orq), Santiago Marín (dir co), Vicente Bianchi (dir inv). Santiago de Chile: Oveja Negra, 2006.

[O-115] A La Tirana, 2009, orq: 2 fl, 2 ob, 2 cl, 2 fg, 2 cor, 2 trp, 3 tbn, tba, timp, pla, tr, tamb, arp, vln I, vln II, vla, vlc, cb. Dur. 5'50", Fon: Grabado el 2/11/2016 en el Teatro Municipal de Nuñoa. Orquesta de Cámara de Chile. Alejandra Urrutia (dir). Inédito. Ed. Transcripción digital de Celso Torres Mora, 2009.

[O-116] Farewell, 2016, V y pf, Dur. 4'40”, Text. Pablo Neruda, Fon: CD Chile fértil. Vicente Bianchi (dir). Santiago de Chile: Autoedición, 2016.

[O-117] Chile fértil, 2016, comx, Dur. 1'30”, Text. La Araucana de Alonso de Ercilla, Fon: CD Chile fértil. Vicente Bianchi (dir). Santiago de Chile: Autoedición, 2016.

[O-118] Alma, no me digas nada, s/f, Text. Juan Guzmán Cruchaga.

[O-119] Himno de la Dirección General de Aeronáutica Civil, s/f, Text. María de los Ángeles Sanhueza.

[O-120] La independencia de Chile, tonada, s/f, VV y ac, Dur. 3'20", Text. Carlos Ulloa Díaz, Fon: LP Música para la Historia de Chile. Silvia Infantas y los Baqueanos. Vicente Bianchi y su Orquesta. Santiago de Chile, EMI-Odeón, 1959 [reed CD, Colección Bicentenario, Santiago de Chile: EMI, 2007].

[O-121] Rin y pericona, s/f, orq: $2 \mathrm{fl}, 2 \mathrm{ob}, 2 \mathrm{cl}, 2 \mathrm{fg}, 2$ cor, 2 trp, 2 tbn, tri, bat, glock/xil, timp, vln I, vln II, vla, vlc, cb. Dur. 4’50”. Ed. Transcripción digital de Sergio Tapia Sandoval.

[O-122] Tradicionales de La Tirana, s/f, pf, Dur. 5'40", Fon: CD Vicente Bianchi con su piano a los 90, Vicente Bianchi (pf), Santiago: Autoedición, 2010, Ed. Vicente Bianchi con su piano a los 90, Santiago: Escuela Moderna de Música y Danza, Sociedad Chilena de Derecho de Autor SCD, 2011.

[O-123] Bailando pericona, s/f, pf, Dur. 3'40", Fon: CD Vicente Bianchi con su piano a los 90, Vicente Bianchi (pf), Santiago: Autoedición, 2010, Ed. Vicente Bianchi con su piano a los 90, Santiago: Escuela Moderna de Música y Danza, Sociedad Chilena de Derecho de Autor SCD, 2011. 